PROCEEDINGS OF THE

AMERICAN MATHEMATICAL SOCIETY

Volume 135, Number 9, September 2007, Pages 2915-2920

S 0002-9939(07)08876-4

Article electronically published on May 9, 2007

\title{
A STRENGTHENING OF THE CARLEMAN-HARDY-PÓLYA INEQUALITY
}

\author{
FINBARR HOLLAND
}

(Communicated by David Preiss)

\begin{abstract}
As a consequence of a more general statement proved in the paper, it is deduced that, if $n \geq 1$, and $a_{j}>0, j=1,2, \ldots, n$, then

$$
\left(\frac{\sum_{j=1}^{n} \sqrt[j]{a_{1} a_{2} \cdots a_{j}}}{\sum_{j=1}^{n} a_{j}}\right)^{1 / n}+\frac{\sqrt[n]{a_{1} a_{2} \cdots a_{n}}}{\sum_{j=1}^{n} \sqrt[j]{a_{1} a_{2} \cdots a_{j}}} \leq \frac{n+1}{n},
$$

with equality if and only if $a_{1}=a_{2}=\cdots=a_{n}$. This is a new refinement of Carleman's classic inequality.
\end{abstract}

\section{INTRODUCTION}

Let $\mathbb{P}$ stand for the group of positive sequences $x: \mathbb{N} \rightarrow(0, \infty)$ under the operation of pointwise multiplication. Fix $w \in \mathbb{P}$, and let $s$ be the corresponding sequence of partial sums defined by $s_{i}=w_{1}+w_{2}+\cdots+w_{i}, i=1,2, \ldots$ With each $a \in \mathbb{P}$, associate the sequence $g(a)$ of weighted geometric means of $a$ defined by

$$
g_{i}(a)=a_{1}^{w_{1} / s_{i}} a_{2}^{w_{2} / s_{i}} \ldots a_{i}^{w_{i} / s_{i}}, i=1,2, \ldots .
$$

The title of the paper refers to the following theorem:

Theorem 1.1. For all $a \in \mathbb{P}$,

$$
\sum_{i=1}^{\infty} w_{i} g_{i}(a) \leq e \sum_{i=1}^{\infty} w_{i} a_{i}
$$

In other words, if $a$ belongs to $\ell_{1}(w)$, the weighted $\ell_{1}$-space of complex sequences $z$, for which $\sum_{i=1}^{\infty} w_{i}\left|z_{i}\right|$ is finite, with $\|z\|_{w}=\sum_{i=1}^{\infty} w_{i}\left|z_{i}\right|$ as its norm, then so does $g(a)$, and $\|g(a)\|_{w} \leq e\|a\|_{w}$.

It would appear from Hardy's own account given in [4, that (11) was first discovered by Pólya, who brought it to Hardy's attention. In any event, following a suggestion made to him by Pólya, which he acknowledges in [4, Hardy obtained (1) by applying a limiting process to his ground-breaking result (that he established in the same paper) to the effect that, if $p>1$, then the Cesàro operator $C$ given by

$$
(C f)(x)=\frac{1}{x} \int_{0}^{x} f(t) d t, x>0,
$$

Received by the editors September 23, 2005 and, in revised form, June 8, 2006.

2000 Mathematics Subject Classification. Primary 26D15.

Key words and phrases. Weighted geometric means, Carleman's inequality, convex functions.

(C)2007 American Mathematical Society Reverts to public domain 28 years from publication 2915 
is a bounded linear transformation from $L^{p}(0, \infty)$ to $L^{p}(0, \infty)$, with norm $p /(p-1)$. Curiously, and somewhat ambiguously, Hardy claimed, without proof, that the constant $e$ in (11) is best possible; but, significantly, this remark is not repeated in [5].

In particular, taking $w_{i}=1, i=1,2, \ldots$, we obtain an elegant inequality due to Carleman, which arose in his study of the class of quasi-analytic functions [2], viz., for all $a \in \mathbb{P}$,

$$
\sum_{n=1}^{\infty} \sqrt[n]{a_{1} a_{2} \cdots a_{n}}<e \sum_{n=1}^{\infty} a_{n}
$$

Furthermore, the constant $e$ is certainly the smallest possible here, as can be seen by noting that

$$
\sum_{k=1}^{n} \frac{1}{\sqrt[k]{k !}} \sim e \sum_{k=1}^{n} \frac{1}{k}(n \rightarrow \infty)
$$

in the sense of asymptotic equality.

Not surprisingly, the Carleman-Hardy-Pólya inequality (11), and its antecedent (21), have evoked much interest over the years, and each has been extended in a variety of ways by a host of authors. An account of several interesting proofs of inequality (2), its continuous analogue and various refinements and generalizations of it, enlivened with some enlightening historical remarks, can be found in [3], which also contains an extensive bibliography referencing these investigations. In addition, the beautiful memoir by Bennett [1] contains, inter alia, more information about these inequalities, and further developments of them.

Our interest in the matters discussed here was re-ignited recently by a paper of Kedlaya 8] who derived (11) from a weighted version of another extension of Carleman's inequality that he proved in [7], which had been conjectured by the author [6]. (Subsequently, it came to light that two stronger forms of this had been stated, without proof, much earlier by Nanjundiah [9].)

Kedlaya proved (11) under the (unnecessary) assumption that the sequence $s w^{-1}$ is non-decreasing, clearly unaware of the earlier work by Hardy and Pólya, and expressed surprise that the constant was independent of $w$. This led me to speculate that a much stronger assertion than (11) may be true under this additional monotonicity requirement. The purpose of this paper is to confirm this.

\section{THE MAIN THEOREM}

Theorem 2.1. If $s w^{-1}$ is non-decreasing, then, for all $a \in \mathbb{P}$, and all $n \in \mathbb{N}$,

$$
\left(\frac{\sum_{j=1}^{n} w_{j} g_{j}(a)}{\sum_{j=1}^{n} w_{j} a_{j}}\right)^{w_{n+1} / s_{n}}+w_{n+1} \frac{g_{n}(a)}{\sum_{j=1}^{n} w_{j} g_{j}(a)} \leq \frac{s_{n+1}}{s_{n}} .
$$

Moreover, equality occurs in (44) if and only if $a_{1}=a_{2}=\cdots=a_{n}$.

Conversely, if (41) holds for all $a \in \mathbb{P}$, and all $n \in \mathbb{N}$, then $s w^{-1}$ is non-decreasing.

Proof. Suppose $s w^{-1}$ is non-decreasing. Let $a \in \mathbb{P}$. Fix $n \in \mathbb{N}$, and, for convenience, denote $g_{j}(a)$ by $g_{j}$. As a function of $a_{1}, a_{2}, \ldots, a_{n}$, the expression on the left-hand side of inequality (44) is homogeneous of degree zero, so it's enough to prove that 
the inequality holds when $\sum_{j=1}^{n} w_{j} g_{j}=1$, a condition we now impose. Assuming this, and using the convexity of the function

$$
f(t)=t^{-w_{n+1} / s_{n}}, t \in(0, \infty),
$$

we see that

$$
\begin{aligned}
\left(\frac{\sum_{j=1}^{n} w_{j} g_{j}}{\sum_{j=1}^{n} w_{j} a_{j}}\right)^{w_{n+1} / s_{n}} & =f\left(\sum_{j=1}^{n} w_{j} g_{j} \frac{a_{j}}{g_{j}}\right) \\
& \leq \sum_{j=1}^{n} w_{j} g_{j} f\left(\frac{a_{j}}{g_{j}}\right) \\
& =\sum_{j=1}^{n} w_{j} g_{j}\left(\frac{g_{j}}{a_{j}}\right)^{w_{n+1} / s_{n}} \\
& =\sum_{j=1}^{n} w_{j} \sqrt[s]{g_{j}^{s_{n+1}} a_{j}^{-w_{n+1}}} .
\end{aligned}
$$

But, defining $s_{0}=0=g_{0}-1$,

$$
a_{j}^{w_{j}}=\frac{g_{j}^{s_{j}}}{g_{j-1}^{s_{j-1}}}, j=1,2, \ldots, n,
$$

and so

$$
g_{j}^{s_{n+1}} a_{j}^{-w_{n+1}}=g_{j}^{s_{n+1}-w_{n+1} s_{j} / w_{j}} g_{j-1}^{s_{j-1} w_{n+1} / w_{j}},
$$

whence, bearing in mind that

$$
\frac{s_{n+1}}{w_{n+1}} \geq \frac{s_{j}}{w_{j}}, j=1,2, \ldots, n,
$$

and that

$$
a^{\alpha} b^{1-\alpha} \leq \alpha a+(1-\alpha) b,
$$

whenever $a, b \geq 0$ and $0 \leq \alpha \leq 1$, we deduce that

$$
\begin{aligned}
\sum_{j=1}^{n} w_{j} \sqrt[s_{n}]{g_{j}^{s_{n+1}} a_{j}^{-w_{n+1}}} & =\sum_{j=1}^{n} w_{j} \sqrt[s_{n}]{g_{j}^{s_{n+1}-w_{n+1} s_{j} / w_{j}} g_{j-1}^{s_{j-1} w_{n+1} / w_{j}}} \\
& \leq \frac{1}{s_{n}} \sum_{j=1}^{n} w_{j}\left[\left(s_{n+1}-\frac{w_{n+1} s_{j}}{w_{j}}\right) g_{j}+\frac{s_{j-1} w_{n+1}}{w_{j}} g_{j-1}\right] \\
& =\frac{s_{n+1}}{s_{n}} \sum_{j=1}^{n} w_{j} g_{j}-\frac{w_{n+1}}{s_{n}} \sum_{j=1}^{n}\left[s_{j} g_{j}-s_{j-1} g_{j-1}\right] \\
& =\frac{s_{n+1}}{s_{n}}-w_{n+1} g_{n} .
\end{aligned}
$$

Thus (4) holds.

Next, we consider the case of equality in (4). Evidently, equality holds if $a_{1}=$ $a_{2}=\cdots=a_{n}$. Conversely, if equality occurs for some $n$, and some $a \in \mathbb{P}$, then, in the presence of the normalization $\sum_{j=1}^{n} w_{j} g_{j}(a)=1$, which we're entitled to make, all signs of inequality in statements (5) through to (8), other than in (7), become 
signs of equality, and so, in particular, there is equality in (6). But $f$ is continuous and strictly convex. Hence

$$
\frac{g_{j}}{a_{j}}=\frac{g_{1}}{a_{1}}, j=1,2, \ldots, n,
$$

whence it follows easily that $a_{1}=a_{2}=\cdots=a_{n}$.

Conversely, if (44) holds for all $a \in \mathbb{P}$, and $n \geq 2$, then, setting $a_{i}=1, i \neq 1, a_{n}=$ $x>0$, we have that

$$
\left(\frac{s_{n-1}+w_{n} x^{w_{n} / s_{n}}}{s_{n-1}+w_{n} x}\right)^{w_{n+1} / s_{n}}+\frac{w_{n+1} x^{w_{n} / s_{n}}}{s_{n-1}+w_{n} x^{w_{n} / s_{n}}} \leq \frac{s_{n+1}}{s_{n}}, \forall x>0,
$$

whence, letting $x \rightarrow \infty$, we deduce that

$$
\frac{w_{n+1}}{w_{n}} \leq \frac{s_{n+1}}{s_{n}} .
$$

Thus $s w^{-1}$ is non-decreasing.

This completes the proof.

\section{TWO COROLLARIES AND AN EXAMPLE}

We draw a number of conclusions, the first of which is obtained by taking $w$ to be the identity in $\mathbb{P}$; it yields a new variant of Carleman's inequality for finite sequences.

Corollary 3.1. For all $a \in \mathbb{P}$, and any $n \geq 1$,

$$
\left(\frac{\sum_{j=1}^{n} \sqrt[j]{a_{1} a_{2} \cdots a_{j}}}{\sum_{j=1}^{n} a_{j}}\right)^{1 / n}+\frac{\sqrt[n]{a_{1} a_{2} \cdots a_{n}}}{\sum_{j=1}^{n} \sqrt[j]{a_{1} a_{2} \cdots a_{j}}} \leq \frac{n+1}{n},
$$

with equality if and only if $a_{1}=a_{2}=\cdots=a_{n}$.

Another simple and immediate consequence of inequality (4) follows by dropping the second term from its left-hand side. It provides information about the constant in the generalisation of Carleman's inequality due to Hardy and Pólya.

Corollary 3.2. If $s w^{-1}$ is non-decreasing, $a \in \mathbb{P}$, and $n \geq 1$, then

$$
\sum_{j=1}^{n} w_{j} g_{j}(a)<\left(1+\frac{w_{n+1}}{s_{n}}\right)^{s_{n} / w_{n+1}} \sum_{j=1}^{n} w_{j} a_{j} .
$$

Moreover, if

$$
K=\sup \left\{\frac{s_{n}}{w_{n+1}}: n \in \mathbb{N}\right\}
$$

and $a \in \ell_{1}(w)$, then $g(a) \in \ell_{1}(w)$, and $\|g(a)\|_{w}<c_{K}\|a\|_{w}$, where

$$
c_{K}= \begin{cases}\left(1+\frac{1}{K}\right)^{K}, & \text { if } K<\infty, \\ e, & \text { if } K=\infty .\end{cases}
$$

Note that inequality (9) is false in general. To see this, refer to (3), and choose $n$ sufficiently large so that

$$
\sum_{k=1}^{n} \frac{1}{\sqrt[k]{k !}} \geq 2 \sum_{k=1}^{n} \frac{1}{k}
$$


Define $w_{j}=1, j=1,2, \ldots, n, w_{j+1}=s_{j}, j \geq n$, and take $a_{j}=1 / j, j=1,2, \ldots$. Then, for this $n$, and this $a$,

$$
\sum_{j=1}^{n} w_{j} g_{j}(a) \geq\left(1+\frac{w_{n+1}}{s_{n}}\right)^{s_{n} / w_{n+1}} \sum_{j=1}^{n} w_{j} a_{j},
$$

in opposition to (9). Thus we cannot dispense with the monotonicity condition.

We end with a simple illustrative example.

Example 3.3. Suppose $r>1, a \in \mathbb{P}$, and $\sum_{n=1}^{\infty} r^{n} a_{n}<\infty$. Then

$$
\sum_{n=1}^{\infty} r^{n} \prod_{k=1}^{n} a_{k}^{r^{k-1}(r-1) /\left(r^{n}-1\right)}<r^{1 /(r-1)} \sum_{n=1}^{\infty} r^{n} a_{n}
$$

The constant $r^{1 /(r-1)}$ is best possible.

Proof. We apply the second corollary with $w_{j}=r^{j-1}, j=1,2, \ldots$, so that $s_{j}=$ $\left(r^{j}-1\right) /(r-1), j=1,2, \ldots$, and

$$
\frac{s_{j}}{w_{j}}=\frac{r^{j}-1}{r^{j-1}(r-1)}, j=1,2, \ldots
$$

Clearly, the conditions of the corollary are satisfied and

$$
\sup \left\{\frac{s_{n}}{w_{n+1}}: n \in \mathbb{N}\right\}=\frac{1}{r-1} .
$$

The stated result follows. To see that no smaller constant works, let $a_{j}=r^{1-j}, j=$ $1,2, \ldots, n ; a_{j}=0, j=n+1, n+2, \ldots$ Then $\sum_{j=1}^{\infty} r^{j-1} a_{j}=n$, and a calculation shows that

$$
\begin{aligned}
\sum_{j=1}^{\infty} r^{j-1} \prod_{k=1}^{j} a_{k}^{r^{k-1}(r-1) /\left(r^{j}-1\right)} & =\sum_{j=1}^{n} r^{j-1} \prod_{k=1}^{j} r^{(1-k) k r^{k-1}(r-1) /\left(r^{j}-1\right)} \\
& =r^{1 /(r-1)} \sum_{j=1}^{n} r^{(-j+(r-2) /(r-1)) /\left(r^{j}-1\right)} \\
& \sim r^{1 /(r-1)} n,
\end{aligned}
$$

as $n \rightarrow \infty$. Hence the constant of the inequality cannot be replaced by a smaller number.

In conclusion, I wish to record my thanks to the referee for his/her perceptive remarks, and for drawing my attention to 3 .

\section{REFERENCES}

[1] G. Bennetr, Factorizing the Classical Inequalities, Mem. Amer. Math. Soc. 120 (1996), no. 576, viii+130 pp. MR.1317938 (96h:26020)

[2] T. Carleman, Sur les functions quasi-analytiques, Fifth Scand. Math. Congress (1923), 181196.

[3] M. Johansson, L. E. Persson, A. Wedestig, Carleman's inequality-history, proofs and some new generalizations, J. Inequal. Pure \& Appl. Math. 4 (3), (2003), 1-19. MR2044402 (2005a:26027)

[4] G. H. HARDY, Notes on some points of the integral calculus (LX), Messenger of Math. $\mathbf{5 4}$ (1925), 150-156.

[5] G. H. Hardy, J. E. Littelwood, and G. Pólya, Inequalities, Cambridge University Press, 1934. MR0046395(13:727e) 
[6] F. Holland, On a mixed arithmetic-mean, geometric-mean inequality, Math. Competitions 5 (1992), 60-64.

[7] K. Kedlaya, Proof of a mixed arithmetic-mean, geometric-mean inequality, Amer. Math. Monthly 101 (1994), 355-357. MR1270962 (95b:26022)

[8] K. Kedlaya, A Weighted Mixed-Mean Inequality, Amer. Math. Monthly 106 (1999), 355358. MR.1543452

[9] T. S. NANJUndiah, Sharpening of some classical inequalities, Math Student 20 (1952), 24-25.

Mathematics Department, University College, Cork, Ireland

E-mail address: f.holland@ucc.ie 